\title{
Risk factors for radiogenic cancer: a comparison of factors derived from the Hanford survey with those recommended by the ICRP
}

\author{
K F BAVERSTOCK AND D G PAPWORTH \\ From the MRC Radiobiology Unit, Harwell, Didcot, Oxon, UK
}

ABSTRACT A model for cancer induction in man exposed to low doses of radiation and based on the analysis of a survey of workers from a nuclear fuel processing plant is examined and compared with that adopted by the ICRP to limit risks to radiation workers. It is shown that claims that ICRP has significantly underestimated the risk apply primarily to those exposed in later life, and arise from assumptions regarding the age dependence of sensitivity to radiation which are questionable. A preliminary attempt is made to test the proposed model using the United Kingdom luminiser population. Deaths from cancers in four tissues believed to be sensitive to radiation induced carcinogenesis are examined and the observed number of deaths in the study population is compared with the number predicted by the model. Taken individually, only one of these sites rejects the model but taken together these four sites, which comprise about half the cancer observed in the luminiser population, provide a conclusive rejection of the model.

The survey of nearly 28000 men and women employed at the Hanford nuclear fuel reprocessing plant constitutes one of the major occupationally exposed groups of radiation workers to be analysed for an excess risk of cancer. The results of the analysis were first published in 1977 by Mancuso, Stewart, and Kneale (MSK).' They claimed to show that the risks of radiation received at low dose rates and in low doses were substantially greater than those recommended by the International Commission on Radiological Protection (ICRP) ${ }^{2}$ for the purposes of planning the exposure of workers to radiation and which are still applied today. Direct comparison of the MSK proposals with the ICRP recommendations was complicated by the form of presentation chosen by MSK-namely, in terms of doubling dose and by the claim that the doseresponse relation was not linear. The doubling dose is the dose necessary to double the "natural" or "spontaneous" incidence of a particular malignant disease, and the term relates specifically to the "relative risk" model for radiation carcinogenesis. By contrast the ICRP, which has adopted the convention of assuming a linear dose-response relation, adopts the "absolute risk" model and presents risks in terms of risk coefficients-for example, the numbers of deaths in $10^{\circ}$ exposed people receiving a unit

Received 30 July 1984

Accepted 17 September 1984 dose over a defined period after exposure. After the initial publication from MSK several criticisms of the methodology and interpretation were made. Subsequent years have seen further publications by MSK in which their analyses have been refined. It is not our intention to review these developments since it has been done adequately elsewhere ${ }^{3}$ but rather to examine a model based on their interpretation of the Hanford findings ${ }^{4}$ in which they propose a dose-response relation for radiation induced cancer at low doses. This model is compared quantitatively with that adopted by the ICRP. The comparison is still not straightforward. The doubling dose concept retained by MSK means that risk varies with age (owing to variations in the natural incidence of the malignant disease that is to be doubled). Their proposed dose-response relation is not linear but of the form $D^{0.5}$. Furthermore, MSK have introduced factors for a varying sensitivity with age (apart from that due to variations in natural incidence) and a factor to take account of latency.

\section{MSK model}

The model, as proposed in 1981 , is based on a multifactorial analysis with the following factors controlled: sex, work cohort, age at hire, employment period, and monitoring for internal radiation. Cancers are divided into two categories: type $\mathbf{A}$ cancers 
that are considered by the authors to be radiosensitive - that is, sensitive to induction by radiation; and type $B$ cancers which are considered not to be radiosensitive. Statistical tests to establish whether there is evidence-for a radiation related effect show that in the Hanford population type A cancers are positively correlated with radiation dose and type B cancers negatively correlated. When the same data are compared on a standardised mortality ratio analysis both types of cancers are in deficit compared with expectation from an age and sex matched sample of white Americans. ${ }^{5}$ In the 1981 analysis MSK explain this deficit as being due to the healthy worker effect. ${ }^{4}$ In later publications, however, it is attributed to a social class effect and selective recruitment. ${ }^{67}$

Having established that type A cancers are positively correlated with dose, MSK proceed to fit a number of simple models and to test them by the method of maximum likelihood. The simplest of these models allows for the variation of the assumed doubling dose and a parameter measuring the nonlinearity of dose response. They conclude that a best fit is obtained when it is assumed that mortality depends on $\mathrm{D}^{0.5}$ and the mean doubling dose for type A cancers is $0 \cdot 15$ Gray (Gy).

This basic model is further elaborated by allowing for a variation in sensitivity to radiation induced cancer with age at exposure and incorporating a factor allowing for the latency of the cancer. Best fits are obtained for:*

(a) dose response- $\mathrm{D}^{0.5}$,

(b) doubling dose- 0.15 Gy with $95 \%$ confidence interval 0.02 to $1.50 \mathrm{~Gy}$,

(c) mean latency-25 years, and

(d) effect of age on cancer induction-increased sensitivity with increasing age by a factor $e$ (the base of natural logarithms) for each eight years.

This model may be used prospectively (to estimate deaths from cancer in an exposed population) or retrospectively (to estimate the probability of a given death having been caused by earlier exposure to radiation). In what follows it will be used in a prospective sense-that is, cohorts of persons

\footnotetext{
*In a more recent publication ${ }^{8}$ slight changes to the 1981 model $^{4}$ appear to have been made. The essential features of a curvilinear dose response, increasing sensitivity with age, and allowance for latency have been retained but with small quantitative changes. The increasing sensitivity with age is by a factor of $10 \%$ a year rather than a factor e for each eight years (equivalent to $13 \%$ a year) and the latency factor instead of being a smooth function reaching a maximum of unity at 25 years after exposure is a step function with a value 0 for exposures less than 10 years before death and unity for all other years after exposure.
}

exposed to radiation at certain ages and to certain $\frac{\vec{D}}{2}$ doses will be considered and the expectation for the ? spontaneous incidence of a particular disease and $\overrightarrow{\vec{F}}$ for the radiation induced excess in the years that follow calculated. This entails calculating what MSK call the cancer effective dose-that is, the physical $\frac{\bar{c}}{\bar{D}}$ dose of radiation multiplied by a factor for the age $\frac{\sigma}{\sigma}$ sensitivity. By convention MSK normalise the age $\varnothing$ factor at 1 for age 40 years at exposure. From this $\%$ cancer effective dose a relative risk factor may be $\overrightarrow{0}$ obtained which when modified by the latency factor may be multiplied by the natural mortality of the $\vec{\omega}$ disease appropriate for the age of the cohort and the $\frac{\Omega}{\circ}$ calendar year to give the mortality of radiation $\mathbb{\Phi}$ induced disease for that year. Summed over the years after exposure this yields the total expected $\hat{N}$ numbers of fatal radiation induced disease in the $i$ cohort.

The diseases categorised under type A, radiosen- $\vec{\overrightarrow{ }}$ sitive cancers, are as follows: alimentary tract, 윽 respiratory system, the female breast, lymphoma, $\vec{\overrightarrow{ }}$ myeloma, myeloid leukaemia, other reticuloendothelial cancers, and thyroid cancer. To compare the MSK model with the assumptions made by $\overrightarrow{0}$ ICRP, leukaemia in women has been chosen as an cor example. This disease has well recognised associa- i tions with radiation, has a high mortality, and is not associated with other common carcinogenic factors (such as use of tobacco). Dose-response curves are calculated according to the model for hypothetical $\frac{\nabla}{2}$ cohorts of women aged 20,40 , and 60 at time of irradiation, each cohort assumed to have been 0 given a single exposure in 1955 and been followed up over the following 25 years to 1980 . Table 1 윰 gives the ratio of deaths predicted by the MSK model to those predicted by ICRP. The ICRP estimates are based on a risk coefficient for leukaemia $\exists$ of 20 deaths $/ 10^{\circ} / 0.01 \mathrm{~Gy}$. The comparison is not $\frac{5}{3}$ quite straightforward since the ICRP risk estimates make no allowance for latency. Thus in the highest age group - namely those exposed at age 60 - many will not live to express a cancer induced at the time 옥 of exposure and so the risk is somewhat less than $D$ calculated. Table 1 gives ratios of MSK prediction to ICRP assumptions at various doses. The most not- $N$ able feature of these ratios is the variation of sensitivity with age. For those aged 20 at time of expos- $\tilde{D}$ ure the MSK model predicts fewer deaths from $\underset{\omega}{\omega}$ cancer than does ICRP, even at the lowest doses. For exposure at age 40 the number of deaths pre- 0 dicted by MSK is significantly greater than would be $\overparen{\varnothing}$ assumed by ICRP. For those exposed at age 60 the $\stackrel{\oplus}{+}$ difference between the two models is substantial, $\square$ MSK predicting between 17 and 50 times more deaths than ICRP at doses of 0.5 and $0.05 \mathrm{~Gy}$ respectively. 
Table 1 Ratio of risk from leukaemia predicted by MSK to that predicted by ICRP for hypothetical populations of women irradiated in 1955 at various ages

\begin{tabular}{lllll}
\hline $\begin{array}{l}\text { Age of population at } \\
\text { time of irradiation in } \\
\text { 1955 (years) }\end{array}$ & $\begin{array}{l}\text { Ratio of excess deaths predicted by } \\
\text { MSK over those predicted by ICRP } \\
\text { at doses of }\end{array}$ \\
\cline { 2 - 5 } & 0.05 Gy & 0.10 Gy & 0.20 Gy & 0.50 Gy \\
\hline 20 & 0.46 & 0.41 & 0.34 & 0.22 \\
40 & 6.6 & 4.6 & 3.3 & 2.1 \\
60 & 51 & 36 & 26 & 17 \\
\hline
\end{tabular}

Application of the MSK model to the UK luminiser data

The United Kingdom luminisers are a good example of a population exposed to relatively low doses of radiation by the standards of most other exposed groups (but somewhat high by the standards of present day occupational exposure). The study population numbers 1110 women exposed to doses up to some $0.7 \mathrm{~Gy}$ (average $0.37 \mathrm{~Gy}$ ) over periods of a few months to several years at dose rates ranging from $0.05 \mathrm{~Gy} /$ year to $0.20 \mathrm{~Gy} / \mathrm{year}$. The doses are derived from a number of film badge measurements made on individual luminisers. ${ }^{9}$ Thus the population has been exposed at doses and dose rates only a few times greater than the limits currently recommended by ICRP ${ }^{2}$ and as such provide a suitable population with which to test the MSK model. Once again the analysis was done in a prospective sense. For each individual luminiser, the risk of dying of (say) leukaemia in year $n$ owing to exposure received in an earlier year $i$ is calculated by multiplying the spontaneous death rate from leukaemia in year $n$ in individuals of the appropriate age group by the so called relative risk factor $R$. MSK give a relation for the calculation of $R$; the calculation takes into account the dose $D$ received by the individual in year $i$, her age at the time of exposure, and the latency period $i-n$. The total risk to the individual concerned of dying of leukaemia in year $n$ is the sum of the separate risks due to the exposures received in each year of employment. By summing these risks for all individuals in the luminiser population still alive at the beginning of year $n$, we obtain the predicted number of deaths from leukaemia occurring in year $n$. This calculation is repeated to obtain the predicted deaths in each year from 1961 to 1980 inclusive. The dose $\mathrm{D}$ is derived in the way previously described from the film badges worn by some luminisers." This dose is a good indication of breast dose but not necessarily of other deeper lying tissue doses. Thus for doses to other organs such as the lung, stomach, and bone marrow some allowance for attenuation of dose in superficial tissues is required. The principal source of the radiation is taken to be the paint in the container on the work table. For the purposes of these calculations it has been assumed that this container was some $80 \mathrm{~cm}$ from the film badge worn on the breast pocket and that the $\gamma$-rays from ${ }^{60} \mathrm{Co}$ will give essentially the same depth dose distribution as those from ${ }^{226} \mathrm{Ra}$. Thus from depth dose tables for large fields ${ }^{10}$ it is possible to make approximate estimates of attenuation factors for each tissue. For lung an average depth of about $5 \mathrm{~cm}$ has been assumed (rather than on the body midline since lung tissue is of much lower density and therefore lower attenuating power than other tissue) giving an attenuation factor of $\mathbf{0 \cdot 8}$. For bone marrow an attenuation factor of 0.5 has been based on marrow distribution"1 assuming $10 \%$ to be at a depth of $4 \mathrm{~cm}, 50 \%$ at a depth of $10 \mathrm{~cm}$, and $40 \%$ at $15 \mathrm{~cm}$. The computed ratio is nearer 0.55 but the lower value of 0.5 has been taken since some of this tissue will be off the central axis and thus subject to a somewhat lower dose than that on the central axis. For stomach a factor of $\mathbf{0 . 6}$ is assumed based on a

Table 2 Observed, expected, and predicted deaths for leukaemia, lung cancer, breast cancer, and stomach cancer in the luminiser population from 1961 to 1980 based on the MSK model and on SMR analysis with the MYCL program and national statistics for England and Wales

\begin{tabular}{|c|c|c|c|c|c|c|c|}
\hline Disease & $\begin{array}{l}\text { Expected deaths } \\
\text { due to natural } \\
\text { incidence }(E)^{*}\end{array}$ & $\begin{array}{l}\text { Predicted deaths } \\
\text { by MSK } \\
\text { analysis }(P)\end{array}$ & $\begin{array}{l}\text { Excess deaths } \\
\text { due to radiation } \\
(=P-E)\end{array}$ & $\begin{array}{l}\text { Observed deaths } \\
\text { in luminiser } \\
\text { population }(O)\end{array}$ & $P / E$ & $O / E \dagger$ & $O / P \dagger$ \\
\hline \multirow{5}{*}{$\begin{array}{l}\text { Leukaemia } \\
\text { (ICD 204-207 inc) } \\
\text { Lung cancer } \\
\text { (ICD 162-163 inc) } \\
\text { Breast cancer } \\
\text { (ICD 174) } \\
\text { Stomach cancer } \\
\text { (ICD 151) } \\
\text { Total }\end{array}$} & $1 \cdot 10$ & 1.89 & 0.79 & 0 & 1.71 & \multirow{5}{*}{$\begin{array}{l}0 \\
(0.33) \\
1.07 \\
(0.48) \\
1.38 \\
(0.11) \\
0.65 \\
(0.41) \\
1.14 \\
(0.25)\end{array}$} & \multirow{5}{*}{$\begin{array}{l}0 \\
(0.15) \\
0.56 \\
(0.072) \\
0.73 \\
(0.11) \\
0.31 \\
(0.04) \\
0.60 \\
(0.0023)\end{array}$} \\
\hline & $6 \cdot 53$ & $12 \cdot 43$ & $5 \cdot 90$ & 7 & 1.90 & & \\
\hline & $13 \cdot 05$ & $24 \cdot 50$ & $11 \cdot 45$ & 18 & \multirow{2}{*}{$\begin{array}{l}1 \cdot 88 \\
(0 \cdot 11) \\
2 \cdot 13\end{array}$} & & \\
\hline & 3.06 & 6.52 & $3 \cdot 46$ & 2 & & & \\
\hline & $23 \cdot 74$ & $45 \cdot 34$ & $21 \cdot 60$ & 27 & 1.91 & & \\
\hline
\end{tabular}

*Calculated using MYCL.

tp-values (on a one tailed exact test) are given in brackets below ratios. 
Table 3 Observed, expected, and predicted deaths for breast cancer subdivided by age and radiation dose for the luminiser population from 1961 to 1980 based on the MSK model and on SMR analysis with the MYCL programme and national statistics for England and Wales

\begin{tabular}{|c|c|c|}
\hline & $\begin{array}{l}\text { Age }<30 y \\
\text { Dose }<0.2 \text { Gy }\end{array}$ & $\begin{array}{l}\text { Age }<30 y \\
\text { Dose } \geqslant 0.2 \text { Gy }\end{array}$ \\
\hline \multirow{3}{*}{$\begin{array}{l}\text { Observed deaths }(\mathrm{O}) \\
\text { Expected deaths due to } \\
\text { natural incidence }(\mathrm{E})^{*} \\
\text { Predicted deaths by } \\
\text { MSK analysis (P) } \\
\text { O/E† } \\
\text { O/P† }\end{array}$} & 2 & 13 \\
\hline & 1.94 & 6.95 \\
\hline & $\begin{array}{l}2.527 \\
1.03(0.58) \\
0.79(0.54)\end{array}$ & $\begin{array}{l}11.612 \\
1.87(0.026) \\
1 \cdot 12(0.38)\end{array}$ \\
\hline \multirow{3}{*}{$\begin{array}{l}\text { Observed deaths }(\mathrm{O}) \\
\text { Expected deaths due to } \\
\text { natural incidence }(\mathrm{E})^{*} \\
\text { Predicted deaths by } \\
\text { MSK analysis (P) } \\
\text { O/E† } \\
\text { O/P† }\end{array}$} & $\begin{array}{l}\text { Age } \geqslant 30 y \\
\text { Dose }<0.2 \text { Gy } \\
2\end{array}$ & $\begin{array}{l}\text { Age } \geqslant 30 y \\
\text { Dose } \geqslant 0.2 \text { Gy } \\
1\end{array}$ \\
\hline & 1.62 & $2 \cdot 56$ \\
\hline & $\begin{array}{l}3.155 \\
1.23(0.48) \\
0.63(0.39)\end{array}$ & $\begin{array}{l}7 \cdot 207 \\
0 \cdot 39(0.28) \\
0 \cdot 14(0.006)\end{array}$ \\
\hline
\end{tabular}

*Calculated using MYCL program.

†Figures in brackets are $p$ values on exact one sided tests.

midline dose. A parallel SMR analysis of the luminiser data using the conventional technique for computing deaths with the man-years computer language (MYCL) was also carried out over the same period. The results are presented in table 2 .

In a previous analysis of the luminiser survey up to 1978 the population was subdivided into two dose groups": those receiving less than $0.2 \mathrm{~Gy}$ and those receiving $\mathbf{0 . 2}$ or more Gy and into two age groups: those under age $\mathbf{3 0}$ and those aged $\mathbf{3 0}$ or more at time of first exposure. This method of analysis showed that the observed excess of breast cancer lay in the group receiving the higher dose but who were in the lower age group at the time of irradiation. Table 3 shows a similar analysis for the MSK model compared with that using the MYCL programme for the period 1961-80.

\section{Discussion}

It is clear from the analysis of the hypothetical populations of uniform age assumed to have been irradiated in 1955 that age at time of irradiation is an overwhelmingly important factor in the MSK model. Cancer rates generally increase with age, so when the doubling dose concept is applied the risk must increase with age. The MSK model, however, also incorporates a further age factor by increasing the sensitivity to cancer induction by a factor e for every eight year increment in age. Thus those irradiated in their later years of life are assumed to be at substantially greater risk than those irradiated early on. Since there is reasonable agreement between MSK predictions and ICRP risk estimates for young $\frac{3}{0}$ adults the validity of this age sensitivity assumption is crucial to the arguments which claim that the $\vec{\Rightarrow}$ ICRP significantly underestimates the risk of radia- $\stackrel{\oplus}{+}$ tion induced cancer at low doses. Evidence from other sources for an influence of age on radiation $\overline{\bar{\sigma}}$ induced cancer is equivocal. In animals radiation $\frac{\bar{\rho}}{\vec{\sigma}}$ sensitivity to leukaemia induction decreases with $\stackrel{\unrhd}{\varrho}$ age $^{12}$; however, this observation may be influenced by the interaction of latency and life span. From $\vec{P}$

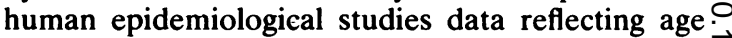
dependence are relatively sparse. Leukaemia $\vec{\omega}$ observed among the atomic bomb survivors shows a $\stackrel{\circ}{\circ}$ minimum sensitivity between ages 10 and 20 in the ${ }_{\Phi}$ absolute risk model with increasing sensitivity up to 3 age 60 by a factor of about 4 by comparison with $20 \mathrm{~N}$ year olds. A similar increase was reported for the $u$ British spondylitics' ${ }^{12}$; however, more recent results $\omega_{\perp}^{\omega}$ from this survey ${ }^{13}$ might indicate that the leukaemia $\overrightarrow{-}$ observed in this group results more from the disease 9 than the treatment since the data show no clear indi- cation of a dose-response relation. Mean doses to the Japanese bomb survivors were probably of the order of $1 \mathrm{~Gy}$ and so the factor $\sim 4$ increase that is $\overrightarrow{0}$ observed should be compared with the factor 17 that or may be derived from table 1 . In fact it is notable that $\square$ in terms of the relative risk model the survivors of the Japanese bomb show no difference in sensitivity between those aged 20 and those aged 60 but that those aged 40 are less sensitive than either by $a \frac{\circ}{\varnothing}$ factor of about 2. ${ }^{14}$ A similar lack of increased sensitivity with age over the age range $20-60$ on the relative risk model is also evident for all other cancers taken together. These data therefore do not? support the hypothesis that sensitivity to radiation induced carcinogenesis increases with age more ${ }_{\mathbb{Q}}$ rapidly than the natural mortality from the given $\supset$ disease, at least at doses of the order of $1 \mathrm{~Gy}$. In $\frac{\mathrm{O}}{3}$ their publications MSK give no mechanistic reason: why such an age sensitivity factor might be thought $ᄋ$ to apply. They derive it from the Hanford data set $₹$ by a process of trial and error in which they deter-윽 mine the maximum likelihood parameter for differ- $\rightarrow$ ent intervals in years by which sensitivity increases by a factor e.

The United Kingdom luminiser population is composed mainly of people who were teenagers or 0 in their early $20 \mathrm{~s}$ at the time of exposure and does not therefore contain a high proportion of those $\sigma$ predicted to be at highest risk by the MSK model. It 0 is, however, a particularly valuable population in view of the comparatively small doses received by? individuals. Between 1961 and 1980 there have $\square$ been 56 deaths from cancer compared with 52 움 expected on the basis of comparison with age and $\overparen{\Omega}$ sex adjusted populations of England and Wales. For 
four cancers classified by MSK as type A which total 27 of the observed deaths from cancer in the luminiser study - namely, leukaemia, lung cancer, breast cancer, and stomach cancer-the ratio of the numbers of predicted deaths with and without exposure to radiation $(\mathrm{P} / \mathrm{E})$ for the luminiser population by the MSK model is about two, whereas the ratios of observed to expected deaths $(\mathrm{O} / \mathrm{E})$ are $0,1 \cdot 03,1 \cdot 38$, and 0.65 for leukaemia, lung cancer, breast cancer, and stomach cancer respectively (table 2 ). Comparison of the number of cancers observed with those predicted by MSK $(\mathrm{O} / \mathrm{P})$ indicates that the deficit is just significant for cancer of the stomach $(p=0.04)$ but not for lung cancer $(p=0.07)$ or breast cancer $(p=0 \cdot 11)$. For leukaemia there are insufficient numbers from which to draw conclusions.

An excess of mortality from breast cancer has already been reported for the luminiser population"; however, the disease is not significantly in excess in the study population as a whole, rather the excess is confined to the subgroup of women under 30 at time of exposure who received breast doses in excess of $0 \cdot 2 \mathrm{~Gy}$. Table 3 shows that MSK would predict $11 \cdot 6$ cancers in this group whereas 13 are observed against 6.95 expected from spontaneously occurring disease. In the remaining three groups - that is, the older women and the low dose group of under 30 year olds-MSK consistently predict higher numbers than are observed. Taking these three groups together, the overall observed numbers of breast cancers are found to be significantly in deficit compared with the number predicted by the MSK model $(p=0.01)$. There is, therefore, unequivocal evidence that the MSK model does not apply to breast cancer. This is in fact not an unexpected finding since it is well known that women over about 40 seem to have a relatively low sensitivity to radiation induced breast cancer. ${ }^{12}$

For lung cancer the position is not so clear cut since this disease is closely associated with smoking. No excess has been observed in the luminiser population where seven cases of lung cancer have been observed and 6.53 are expected. On the basis of the ICRP risk factor ${ }^{2}$ (20 deaths $/ 10 \% 0.01 \mathrm{~Gy}$ ), and assuming an average lung dose of about $0.3 \mathrm{~Gy}$, one extra case may be expected.

Cancer of the stomach is classified as type $\mathrm{A}$ by MSK but is not separately identified in the list of weighting factors given by ICRP. ${ }^{2}$ It is, however, mentioned as one of the tissues thought to be sensitive to radiation induced carcinogenesis which together account for $30 \%$ of the whole body riskno single tissue exceeding a risk factor of 10 deaths $/ 10 \% / 0 \cdot 01 \mathrm{~Gy}$. On the basis of this risk factor and the average dose to the stomach (of about 0.2 Gy) not more than one extra case would be expected in the survey population.

\section{Conclusion}

For cancers of the breast, lung, and stomach taken individually this test indicates little more than a lack of support for the MSK model. The model was derived, however, not for single sites but for the group of type A cancers taken together. The four sites examined in this paper, when taken together, provide a conclusive rejection of the model $(p=$ $0 \cdot 002$ ). These four sites account for about half the total cancer so far observed in the luminiser survey, and this yield is close to that expected on the basis of comparison with the age and sex adjusted population of England and Wales $(\mathrm{O} / \mathrm{E}$ for all neoplasms = 1.08). In fact all the excess in cancer may be accounted for by the excess in breast cancer among women aged under 30 at exposure.

\section{References}

' Mancuso TF, Stewart AL, Kneale GW. Radiation of Hanford workers dying from cancer and other causes. Health Phys 1977;33:369-85.

${ }^{2}$ ICRP. Recommendation of the International Commission on Radiological Protection. Annals of ICRP 1977;1. (ICRP publication 26.)

${ }^{3}$ Darby SC, Reissland JA. Low levels of ionising radiation and cancer-are we underestimating the risk? Journal of the Royal Statistical Society 1981;144:300-14.

${ }^{4}$ Kneale GW, Mancuso TF, Stewart AL. Hanford radiation study III: a cohort study of the cancer risks from radiation to workers at Hanford (1944-77 deaths) by method of regression model in life-tables. Br J Ind Med 1981;38:156-66.

${ }^{s}$ Gilbert ES, Marks S. An analysis of mortality of workers in a nuclear facility. Radiat Res 1979; 79:122-48.

${ }^{6}$ Kneale GW, Mancuso TF, Stewart AL. Job related mortality risks of Hanford workers and their relation to cancer effects of measured doses of external radiation. In: Biological effects of low level radiation: proceedings of a symposium, Venice 1115th April 1983. Vienna: International Atomic Energy Authority, 1983.

' Kneale GW, Mancuso TF, Stewart AM. Identification of occupational mortality risks for Hanford workers. $\mathrm{Br} J$ Ind Med 1984;41:6-8.

${ }^{8}$ Kneale GW, Mancuso TF, Stewart AM. Job related mortality risks of Hanford workers and their relation to cancer effects of measured doses of external radiation. $\mathrm{Br} \mathrm{J}$ Ind Med 1984;41:9-14.

' Baverstock KF, Papworth DG, Vennart J. Risks of radiation at low dose rates. Lancet $1981 ; \mathrm{i}: 430-3$.

${ }^{10}$ British Institute of Radiology. Central axis depth doses data for use in radiotherapy. Br J Radiol 1972;suppl 11.

" Jones TD. CHORD Operators for cell-survival models and insult assessment to active bone marrow. Radiat Res 1977;71:26983.

12 Committee on the Biological Effects of Ionising Radiation. The effects on population of exposure to low levels of ionising radiation. Washington DC: National Academy Press, 1980.

${ }^{13}$ Smith PG, Doll R. Mortality among patients with ankylosing spondylitis after a single treatment course with $\mathrm{x}$ rays. $\mathrm{Br} \mathrm{Med}$ J 1982;284:449-60.

14 Beebe GW, Kato H, Land CE. Studies of the mortality of A-bomb survivors. 6. Mortality and radiation dose 19501974. Radiat Res 1978;75:138-201. 